\title{
Editorial
}

\section{Materials Science of Bioceramic Coatings: An Editorial}

\author{
Robert B. Heimann
}

\section{Am Stadtpark 2A, D-02826 Görlitz, Germany}

Presently, the development of novel ceramic materials with improved biomedical functions is at the forefront of health-related issues in many countries. Arguably, research into bioceramics including coatings for endoprosthetic implants has reached a level of involvement and sophistication comparable only to developments ongoing in the realm of electronic ceramics [1]. Despite the fact that calcium phosphate-based coatings deposited on hip, knee and dental implants as well as bone screws and osteosynthetic devices have an impressive history of clinical success, the quest for improving the longevity of implants and to impart them with better physiological properties is high up on the agenda of numerous research groups around the world. The contributions in this topical issue of The Open Biomedical Engineering Journal attest to these developments.

Until recently, calcium phosphate coatings were deposited onto the surfaces of metallic implants under conditions that were highly non-physiological, involving high temperature technologies such as atmospheric plasma spraying that led to severe thermal decomposition of the precursor hydroxyapatite powders. Although calcium phosphate coatings improve the osseoconductivity of metal implants, they do not render them osseoinductive per se. Indeed, osseoinductive properties can only be conferred onto them by adding an osteogenic growth factor into the system. On the one hand, osteoconductivity is the ability of a biomaterial to foster the in-growth of bone cells, blood capillaries, and perivascular tissue into the gap between implant and existing bone. On the other hand, osteoinduction refers to the transformation of undifferentiated mesenchymal precursor stem cells into osseoprogenitor cells that precede ossification either orthotopically, i.e. within the bony surrounding or hetero- or ectopically, i.e. within muscle or fatty tissues.

The quest of rendering calcium phosphate implant coatings osseoinductive is one of the innate problems to be solved during research required for the next generation of implants. To reach this goal, great effort is being expended, aimed at functionalizing such coatings with osteogenic agents. This includes the use of bone morphogenetic protein2 (BMP-2) and several other non-collagenous proteins and biopolymers that have been proven to confer potent

*Address correspondence to this author at the Am Stadtpark 2A, D-02826

Görlitz, Germany; E-mail: robert.heimann@ocean-gate.de

${ }^{\S}$ Guest Editor osseoinductive properties onto calcium phosphate coatings [2], even triggering ectopic bone formation. However, the contribution by Kokubo and Yamaguchi contained in this special topical issue of TOBEJ charts a way towards a new understanding of the responses of metallic implant materials, in particular titanium and some of its alloys to the biological environment within the human body. As these authors have experimentally shown, the titanium metal surface can be conditioned by exposure to strongly acidic or alkaline solutions and subsequent heat treatment so that the large Zeta potentials established are able to attract $\mathrm{Ca}^{2+}$ and/or $\mathrm{PO}_{4}{ }^{3-}$ ions from simulated body fluid, forming an apatite film with decidedly osseoinductive properties (see below). While these findings will have important bearings on further research into this field, they may be somewhat overenthusiastic as there is often too little scientific evidence to support claims of the efficacy of surface-treated titanium implants to trigger bone neoformation in humans.

However, commercial deposition of bioceramic coatings is still largely been done by thermal technologies, in particular plasma spraying. Indeed, this is still the state-ofthe-art method of choice selected by industry, owing to its economy of operation even though it is a line-of-sight technique. There is a drawback, however, since the high temperature process of plasma spraying leads to incongruent melting and thus thermal decomposition of hydroxyapatite towards tricalcium phosphate (TCP), tetracalcium phosphate (TTCP), and highly cytotoxic calcium oxide $(\mathrm{CaO})$. Additional intermediate phases such as oxyhydroxyapatite (OHAp), oxyapatite (OAp), and amorphous calcium phosphate (ACP) with solubility different from those of hydroxyapatite frequently occur and may influence the biological performance of biomedical coatings. Some of these compounds will interact with body fluid to generate secondary calcium-deficient and thus non-stoichiometric nano-structured hydroxyapatite, a.k.a. bone-like apatite that will act as a template for in-growth of bone cells to anchor an implant solidly to the cortical bone matter. Knowledge of this complex transformation sequence is essential to design coatings with optimum mechanical and chemical long-term stability as well as those properties that will guarantee superior biological performance in vivo.

Tadashi Kokubo and Seiji Yamaguchi of Chubu University, Japan revealed general principles governing the quest for obtaining bone-bonding osseoconductive titanium implants. Systematic studies showed that a suitable combination of surface treatment of $\mathrm{Ti}$ implants with either 
strongly acidic or alkaline solutions, followed by appropriate heat treatment, leads to conditions conducive to the formation of an apatitic surface layer upon exposure to simulated body fluid (SBF). It was thus ascertained that apatite formation entirely rests on surface charge. In particular, soaking of Ti surfaces in $\mathrm{HCl}$ results in a titanium oxide film with a strongly positive Zeta potential whereas treatment with $\mathrm{NaOH}$ forms a sodium titanate film with strongly negative Zeta potential. Owing to formation of a thin apatite layer, the Ti implants tend to bond tightly to the surrounding cortical bone matter. Porous $\mathrm{Ti}$ implants perform particularly well. When exposed to strongly acidic or alkaline solutions, and subsequently heat-treated, they show not only osseoconductive but also osseoinductive functionality. However, one might argue that the presence of a surface charge per se does not necessarily lead to secondary apatite formation as an uncharged hydroxyapatite particle would also become coated with hydroxyapatite if the incubating liquid is supersaturated as in SBF. In contrast to this objection, Kokubo et al. [3] maintain that surface charge is an important precondition for the bone bonding mechanism of hydroxyapatite.

Within this study, it was also established that some $\mathrm{Ti}$ alloys manipulated with the aim to sensitize their surfaces towards formation of a bone-bonding apatite film do not perform in the expected way. For example, some Ti alloys form apatite films only after surficial removal of alloying elements such as $\mathrm{Al}, \mathrm{V}$ or $\mathrm{Nb}$ with a strong solution of $\mathrm{NaOH}$, and subsequent soaking in a strongly acidic solution and associated heat treatment. Other Ti alloys displayed apatite formation only when subjected to a $\mathrm{CaCl}_{2}$ treatment after $\mathrm{NaOH}$ treatment, forming Ca-deficient calcium titanate at their surfaces after subsequent annealing and hot water treatment. This calcium titanate film appears to trigger precipitation of hydroxyapatite since there is an epitaxial structural relation between the (022) lattice plane of calcium titanate (perovskite) and the (00.1) plane of hydroxyapatite [4]. Numerous animal model (rabbit) studies were performed by Kokubo et al. to establish the efficacy of surface-treated titanium implants to trigger new bone formation. However, these results may give an exaggerated bone forming response that frequently cannot be observed in humans. Hence, as a next step the experimental results need to be statistically supported by clinical data even though bioactive Ti implants subjected to $\mathrm{NaOH}$ and heat treatments are already clinically used as an artificial hip joint material in Japan since 2007. Porous $\mathrm{Ti}$ implants subjected to $\mathrm{NaOH}, \mathrm{HCl}$ and heat treatments have successfully undergone clinical trials as spinal fusion devices.

Since the mid-1980s, plasma-sprayed hydroxyapatite coatings are being successfully applied to implant devices. Indeed, plasma spraying has evolved as the most developed, economic, and successful treatment of metallic, in particular Ti-based, implants used for fast integration to bone tissue. However, as mentioned above plasma spraying always induces the formation of dehydroxylation and decomposition phases. In this context, formation of amorphous calcium phosphate (ACP) is an important intervention as crystallinity of a HAp coating is considered a crucial factor for long-term coating stability in vivo. Hence, there is a desire to increase the degree of crystallinity of plasma-sprayed HAp coatings that usually lies between 65 and 85 vol. percentage. To limit the amount of easily soluble non-apatitic calcium phosphate phases, the steep temperature gradient prevailing during plasma spraying has to be reduced by both increasing the substrate temperature and controlling the cooling rate and/or by limiting the thermal transfer from the plasma plume to the powder particles. Imane Demnati et al. of a research team led by Christian Rey of Université de Toulouse, France pursued the option to control the degree of melting of the feedstock powder by low-energy plasma spraying (LEPS). The low heat input into the plasma torch compared to conventional atmospheric plasma spraying avoids overheating and hence thermal decomposition of the powder particles as well as excessive local overheating of the coating/substrate. In addition, LEPS also allows depositing highly porous $(\sim 20 \mathrm{vol} . \%)$ and thick coatings $(250 \mu \mathrm{m})$ without accumulation of deleterious residual tensile stresses that are known to be detrimental to coating performance as they promote formation of cracks, and thus accelerate dissolution in vivo. Another strategy to overcome the decomposition of HAp and thus to limit the presence of soluble non-apatitic calcium phosphate phases is to utilize apatitic ceramics with higher thermal stability such as fluorapatite (FAp) or chlorapatite (ClAp). Indeed, the authors demonstrated that both materials lead to coatings with superior adhesive strength to the substrate as well as significant thermal stability and osseoconductivity.

The objective of the study by Demnati et al. was to compare chlorapatite and hydroxyapatite coatings deposited by LEPS technology. The authors describe a method for facile preparation of chlorapatite from commercial HAp preserving the powder characteristics of the latter. They compare major physico-chemical and mechanical properties of the coatings obtained by the low-energy plasma spraying process, using standardized procedures and specific techniques such as Raman microscopy mapping of the main mineral phases found in the coating, and mechanical testing using three-points bending. A problem might be the need to overcome the low adhesion strength of both types of coating of only $9 \mathrm{MPa}$ that will not stand up to the clinical requirements yet. Other subjects to be addressed in the future will concern the cell metabolical effect of chloride ions released from ClAp coatings, the determination of the bioactivity of the coatings in vitro as well as the evaluation of the bone bonding properties in vivo. Presently, these properties are being studied in animal models as a next step towards possibly establishing this novel type of coating in the clinical praxis.

Along the same line of reasoning, Arjun Dey and Anoop Kumar Mukhopadhyay used a low-power microplasma spraying method (MIPS) to coat HAp onto SS316L substrates to minimize the problems associated with commercial APS HAp coatings. It has now been realized, that conventional high-power plasma spraying creates several disadvantages in terms of phase impurity, reduced porosity limiting osseointegration, and residual stresses, all of which ultimately lead to inadequate mechanical properties and delamination of the coating. Further, the limited crystallinity of HAp deposited by APS accelerates the rate of resorption in vivo that may cause poor adhesion due to a fast mass loss of the HAp coatings. In their contribution, the 
authors used surgical-grade austenitic $316 \mathrm{~L}$ stainless steel as the substrate material because it is more cost effective than Ti6Al4V and CoCrMo alloys, an important consideration in a country such as India with a high proportion of less affluent patients. Phase-pure, highly crystalline HAp coatings were deposited on SS316L substrates by microplasma spraying (MIPS) and were comprehensively characterized. In spite of their heterogeneous microstructure with inter/intra-splat microcracks and $\sim 50-70 \mu \mathrm{m}$ sized macro- and $\sim 1 \mu \mathrm{m}$ sized micropores, as well as 20 vol.\% open porosity, the coatings exhibited moderate adhesive bond strength of $\sim 13 \mathrm{MPa}$ as well as high nano-hardness (6.2 GPa) and Young's modulus (92.3 GPa). Nanoindentation-induced crack length measurement resulted in a stress intensity value $K_{\mathrm{Ic}} \sim 0.60 \mathrm{MPa} \cdot \sqrt{\mathrm{m}}$.

A completely different approach to bioceramic coatings with desirable osseoconductive functionality consists in biomimetic deposition of calcium phosphate films and coatings. Biomimetics is a distinct and rapidly growing discipline that provides insight into the secrets how Nature's biological pathways work, how they are manifest in diverse aspects of chemistry, physics and engineering, and in which way they can be emulated to provide materials and objects useful for biomedical applications including implant coatings.

This novel and promising philosophy of biomimetics impacts much on the contemporary science and engineering of biomedical implants. In Nature, all living organisms utilize proteins, glycoproteins or biopolymers such as polysaccharides as structure-mediating templates to build supporting and protecting mineral structures such as shells, bone, and teeth. Biopolymers control the growth rate, phase composition, crystallite size and morphology, and crystallite orientation of biominerals based on calcium carbonate (calcite, aragonite, vaterite) in mollusc shells or hydroxyapatite in teeth and bones of vertebrae [5]. Similarly, research is being conducted to exploit Nature's propensity to build many hard tissue types by emulating natural mineralization processes and to apply their strategies to improve the structure and properties of bioceramic coatings. Solution-based processes have been developed that mimic natural mineralization pathways via template-mediated selforganisation. These processes involve tightly controlled crystal nucleation and growth as well as controlled phase formation. By using these strategies, uniform coatings can be applied to almost any surface, preferentially porous surfaces that provide easy access to aqueous solutions from which coatings can be grown. Incorporation of osteogenic agents such as non-collagenous proteins into biomimetically deposited coatings can induce strong osseoinductive behaviour [6-8].

Hence, the biomimetic coating method is designed to trigger nucleation and growth of bone-like hydroxyapatite crystals on metallic, ceramic and polymer substrates irrespective of their surface structure and geometry by immersion in a supersaturated solution of calcium phosphate under near-physiological conditions in terms of temperature and $\mathrm{pH}$ to generate self-assembled monolayers (SAMs) [9]. Biomimetic calcium phosphate coatings are now being developed for bone regeneration and repair owing to their biocompatibility, osseoconductivity, and ease of preparation under physiological conditions. Moreover, they can be rendered osseoinductive by incorporating an osteogenic agent, such as bone morphogenetic protein 2 (BMP-2) into the crystalline latticework under physiological situations. Biomimetic calcium phosphate coatings enable a controlled, local and slow release of BMP-2 when it undergoes degradation induced by osteoclasts, that mimics a physiologically-like release mode, to achieve sustained ectopic or orthotopic bone formation. Therefore, biomimetic calcium phosphate coatings are considered a promising delivery vehicle for osteogenic agents or drugs. Low temperature processing as demonstrated by biomimetic deposition of calcium phosphates maintains the chemical integrity of non-collagenous proteins added to the coatings to render them osseoinductive. This is a decisive advantage over high temperature deposition such as thermal spraying.

Along these lines, Klaas de Groot $e t$ al. of University of Amsterdam, The Netherlands provided an overview of biomimetic calcium phosphate coatings including their preparation techniques, physico-chemical properties, potential as drug carriers, and pre-clinical application both in ectopic and orthotopic animal models. Furthermore, they briefly reviewed some features of hydroxyapatite coatings and their clinical applications, in order to gain insights into the clinical applications of biomimetic calcium phosphate coatings in the near future. The biomimetic technique to generate layers of calcium phosphate can be applied to implant coatings biologically functionalized with the osteogenic agent BMP-2. The ultimate aim of this research is to develop a slow-release delivery system for osteogenic agents to promote local bone formation around dental or orthopedic implants, and thereby speeding up their osseointegration. Since the process is entirely conducted under physiological conditions of temperature and $\mathrm{pH}$, protein molecules such as BMP-2 can be and have been incorporated into the inorganic crystal latticework at the time of its deposition. When the inorganic components of the coating are dissolved, the protein molecules remain as a selfsupporting scaffold, which can be remineralized. The incorporated protein enhances the mechanical strength of the coating necessary to withstand the high shear forces generated during surgical implantation.

The slow release of BMP-2 incorporated in a coating has been confirmed by the authors and its osseoinductive potency established when incorporated into calcium phosphate coatings both in vitro and in vivo. Currently, de Groot et al. study the possibility of biomimetic calcium phosphate coating and functionalizing bone substitutes for filling and repairing large osseous defects. In addition, a clinical trial is now underway for treatment of some specific cases involving repairing a bone defect.

Anna Zykova et al. from Kharkov, Ukraine present an account on a less frequently applied, but nevertheless rather promising technology to deposit hydroxyapatite coatings at low temperature. Since the deposition conditions greatly influence the structure and biofunctionality of calcium phosphate coatings, their research has focused on developing advanced methods to deposit double-layered ceramic oxide (alumina)/calcium phosphate coatings by a hybrid technique 
involving r.f. magnetron sputtering and thermal substrate deposition (hydroprocessing) methods. The thermal substrate deposition method is based on the well-known fact that the solubility of hydroxyapatite in water decreases with increasing temperature. Both increasing the concentration of either $\left[\mathrm{Ca}^{2+}\right]$ or $\left[\mathrm{PO}_{4}{ }^{3-}\right]$ or the $\mathrm{pH}$ value, i.e. $\left[\mathrm{OH}^{-}\right]$act to initiate precipitation of hydroxyapatite onto a heated target material. Hence, increasing the temperature of the solution fosters the degree of supersaturation and precipitation of HAp at the heated substrate surface but not in the bulk of the solution or at the confining walls of the experimental vessel. To achieve optimum results it is important to control the ionic concentrations of $\mathrm{Ca}^{2+}$ and $\mathrm{PO}_{4}{ }^{3-}$, the $\mathrm{pH}$, and the temperature to a high degree.

Zykova et al. deposited by this method hydroxyapatite directly at the substrate surface without any precipitation in the initial solution. Using the thermal substrate method, calcium phosphate coatings were prepared at substrate temperatures of $100-105{ }^{\circ} \mathrm{C}$. The coated metallic implant surfaces supplied with a ceramic (alumina) bond coat and a calcium phosphate layer combine the excellent mechanical properties of metals with the chemical stability of a ceramic material, i.e. alumina. Potentiodynamic polarization test results show that the alumina bond coat and the doublelayered alumina-calcium phosphate coatings improve the corrosion resistance compared with uncoated Ti6Al4V and single-layered Ti6Al4V/calcium phosphate substrates. In addition, the double-layered alumina/hydroxyapatite coatings demonstrate the best biocompatibility during in vitro tests on fibroblasts extracted from rat hypodermic cellular tissue and cultivated in Dulbecco's Modified Eagle's Medium (DMEM).

In conclusion, several important aspects of bioceramic coatings, their deposition techniques, properties, and biomedical application as coating for endoprosthetic implants are being addressed in this thematic issue of The
Open Biomedical Engineering Journal. In particular, room is being given to deposition technologies different from conventional high-power thermal spraying, including lowenergy plasma spraying (LEPS), microplasma spraying (MIPS), biomimetic deposition, and thermal substrate deposition (hydroprocessing). In addition, alternative bioceramic materials are being discussed including fluorapatite and chlorapatite that have been shown to be thermodynamically more stable than conventional hydroxyapatite.

\section{REFERENCES}

[1] R. B. Heimann, and H. D. Lehmann, Bioceramic Coatings for Medical Implants. Trends and Techniques. Wiley-VCH, Weinheim, Germany. In the press.

[2] E. Carlisle, and J. S. Fischgrund, "Bone morphogenetic proteins for spinal fusion”, Spine J., vol. 5, no. 6 Suppl, pp. 240S-249S, 2005.

[3] D. K. Pattanayak, S. Yamaguchi, T. Matsushita, T. Nakamura, and T. Kokubo, "Apatite-forming ability of titanium in terms of $\mathrm{pH}$ of the exposed solution", J. R. Soc. Interface, vol. 9, pp. 2145-2155, 2012.

[4] D. Wei, Y. Zhou, D. Jia, and Y. Wang, "Structure of calcium titanate/titania bioceramic composites on titanium alloy and apatite deposition on their surfaces in a simulated body fluid", Surf. Coat. Technol., vol. 201, pp. 8715-8722, 2007.

[5] A. A. Campbell, "Bioceramics for implant coatings", Materials Today, vol. 6, no. 11, pp. 26-30, 2003.

[6] Y. Liu, J. P. Li, E. B. Hunziker, and K. de Groot, "Incorporation of growth factors into medical devices via biomimetic coatings", Phil. Trans. Royal Soc. A, vol. 346, no. 1838, pp. 233-248, 2006.

[7] Y. L. Liu, K. de Groot, and E. B. Hunziker, "Biomimetic mineral coatings in dental and orthopaedic implantology" Front. Mater. Sci. China, vol. 3, no. 2, pp. 154-162, 2009.

[8] Y. L. Liu, G. Wu, and K. de Groot, "Biomimetic coatings for bone tissue engineering of critical-sized defects", J. Royal Soc. Interface, vol. 7, pp. S631-S647, 2010.

[9] B. C. Bunker, P. C. Rieke, B. J. Tarasevich, A. A. Campbell, G. E. Fryxell, G. L. Graff, L. Song, J. Liu, J. W. Virden, and G. L. McVay, "Ceramic thin-film formation on functionalized interfaces through biomimetic processing", Science, vol. 264, no. 5155, pp. $48-55,1994$

This is an open access article licensed under the terms of the Creative Commons Attribution Non-Commercial License (http://creativecommons.org/licenses/ by-nc/3.0/) which permits unrestricted, non-commercial use, distribution and reproduction in any medium, provided the work is properly cited. 